STOCHASTIC MODELING AND CONTROL

BANACH CENTER PUBLICATIONS, VOLUME 122

INSTITUTE OF MATHEMATICS

POLISH ACADEMY OF SCIENCES

WARSZAWA 2020

\title{
REVISITING LINEAR AND LOGNORMAL STOCHASTIC VOLATILITY MODELS
}

\author{
JACEK JAKUBOWSKI and MACIEJ WIŚNIEWOLSKI \\ Institute of Mathematics, University of Warsaw \\ Banacha 2, 02-097 Warszawa, Poland \\ ORCID: 0000-0002-9621-7129, 0000-0001-7025-8275 \\ E-mail: jakub@mimuw.edu.pl, M.Wisniewolski@mimuw.edu.pl
}

\begin{abstract}
In this paper we revisit linear stochastic volatility models with correlated Brownian noises. In such models the asset price satisfies a linear SDE with coefficient of linearity being the volatility process, and a volatility equation with time-dependent coefficients. This class contains among others the Black-Scholes model, the Heston model and the log-normal stochastic volatility model. We present a representation theorem for the density of price, conditions ensuring smoothness of density and some other properties. As an application of using of our general framework we can refine the results for the log-normal stochastic volatility model with correlated noises.
\end{abstract}

1. Introduction. The famous Black-Scholes model with its relatively stringent assumptions does not capture many phenomena of modern financial markets. A prime example is the stochastic nature of the financial asset's volatility, called volatility smile (see for example Hull and White [6]). In recent years many stochastic volatility models have been introduced and developed. However, making the volatility stochastic complicates the models considerably (see for example Rebonato [14]). It is not our aim to review the broad range of stochastic volatility models. We focus on and develop idea of modeling stochastic volatility as SDE with time-dependent coefficients, so in the simplest possible but effective way. We start with a theorem giving a representation of the density function of the underlying asset price in a linear stochastic volatility model. This representation allows us to find a closed formula for the density function, which is important for appli-

2010 Mathematics Subject Classification: Primary 91B25; Secondary 60H30, 91G80.

Key words and phrases: stochastic volatility model, representation, correlated Brownian motions, density function, log-normal stochastic volatility model.

The paper is in final form and no version of it will be published elsewhere. 
cations (see, e.g., Carmona and Durrleman [2]). We formulated the conditions ensuring that the density function of $X_{t}$ belongs to the class $C^{\infty}=\mathcal{C}^{\infty}((0, \infty))$, and then give a form of the density. Moreover, in a few theorems we present some probabilistic properties of density of $X$ in a linear stochastic volatility model, among others an interesting factorization of the density of $\xi_{t}=\frac{X_{t}^{2}}{4 \gamma}$, where $\gamma$ is a gamma random variable with parameter $1 / 2$ independent from $X$. Knowing the moments $\mathbb{E}\left(\ln X_{t}\right)^{n}$ we can compute $\mathbb{E}\left(X_{t} e^{-\lambda X_{t}^{2}}\right)$ under some assumptions, which in fact leads to the closed form of density of $\xi_{t}$. Let us note that some interesting results regarding moments in stochastic volatility models can be found in Lions and Musiela [12].

In the last section we present how using our general framework we can refine the results for specific models. We consider the log-normal stochastic volatility model with correlated noises. Using the representation of density we present forms of density of the price process, which improve the formula presented in Gulisashvili [4]. As an application of our results we obtain a new result for the Hartman-Watson distribution (Corollary 4.5 (other results on the Hartman-Watson distribution can be found in [9]). We also find a recurrence which enables to compute $\mathbb{E}\left(\ln X_{t}\right)^{n}$ for every $n=1,2, \ldots$.

2. Preliminaries. We consider a market defined on a complete probability space $(\Omega, \mathcal{F}, \mathbb{P})$ with filtration $\mathbb{F}=\left(\mathcal{F}_{t}\right)_{t \in[0, T]}, T<\infty$, satisfying the usual conditions. By $\mathbb{F}^{Z}$ we denote the natural filtration generated by a process $Z$ augmented to satisfy the usual conditions. Without loss of generality we assume that the savings account is constant and identically equal to one. Moreover, we assume that the price $X_{t}$ at time $t$ of the underlying asset has a stochastic volatility $Y_{t}$, which has the dynamics given by time dependent SDE, so the dynamics of the vector $(X, Y)$ is given by

$$
\begin{aligned}
d X_{t} & =Y_{t} X_{t} d W_{t}, \\
d Y_{t} & =\mu\left(t, Y_{t}\right) d t+\sigma\left(t, Y_{t}\right) d Z_{t},
\end{aligned}
$$

where $X_{0}, Y_{0}$ are positive constants, the processes $W, Z$ are correlated Brownian motions, $d\langle W, Z\rangle_{t}=\rho d t$ with $\rho \in(-1,1)$, and $\mu: \mathbb{R}_{+} \times \mathbb{R}_{+} \rightarrow \mathbb{R}, \sigma: \mathbb{R}_{+} \times \mathbb{R}_{+} \rightarrow \mathbb{R}$ are continuous functions such that there exists a unique strong solution of (2), which is positive. We have $\int_{0}^{T} Y_{u}^{2} d u<\infty \mathbb{P}$-a.s. since $Y$ is continuous, so it follows from the well known properties of stochastic exponent that a unique strong solution of SDE (1) on $[0, T]$ has the form

$$
X_{t}=X_{0} \exp \left(\int_{0}^{t} Y_{u} d W_{u}-\frac{1}{2} \int_{0}^{t} Y_{u}^{2} d u\right)
$$

(see, e.g., Revuz and Yor [15]). The process $X$ is a local martingale, so there is no arbitrage on such a market. We assume that the asset price is being modeled under the risk-neutral probability.

We call this model a linear stochastic volatility model, because the SDE (1) governing the asset price is linear with respect to the asset price itself with the coefficient of linearity being the stochastic volatility $Y$. Note that the known models such as Black and Scholes model, log-normal stochastic volatility model, Heston model (where $Y^{2}$ is a CIR process) and Stein and Stein model belong to this class. For a review of such models see [4, Chapter 2]. 
REMARK 2.1. It is worth noticing that the constant $\rho$ in the model can be replaced by a measurable, deterministic function $\rho:[0, T] \rightarrow(-1,1)$ and the results of this paper remain true with minor modifications.

3. Existence of the density function and its representation. We start with a theorem on existence of the density function of the underlying asset price in a linear stochastic volatility model, and its representation. This representation allows us to find a closed formula for the density function (see examples in the next section), which is important for applications (see, e.g., Carmona and Durrleman [2]). Of course, our theorem gives for the Black-Scholes model the well-known density function of price. Gulisashvili in [4. Section 3] gives various representations formulas for stock price densities in the specific linear stochastic volatility models. Our technique of proof is different from that in [4].

By $\Phi$ (resp. $\phi$ ) we denote the cumulative distribution function (resp. the density function) of the standard Gaussian random variable.

THEOREM 3.1. Fix $t \in(0, T]$. In a linear stochastic volatility model the distribution of $X_{t}$ has the representation

$$
\mathbb{P}\left(X_{t} \leq r\right)=\mathbb{E} \Phi\left(\frac{\ln \left(r / X_{0}\right)-\mu_{Z}(t)}{\sigma_{Z}(t)}\right),
$$

where $r>0$, and

$$
\begin{aligned}
& \mu_{Z}(t)=\rho \int_{0}^{t} Y_{u} d Z_{u}-\frac{1}{2} \int_{0}^{t} Y_{u}^{2} d u \\
& \sigma_{Z}^{2}(t)=\left(1-\rho^{2}\right) \int_{0}^{t} Y_{u}^{2} d u
\end{aligned}
$$

Moreover, the random variable $X_{t}$ has the density function $g_{X_{t}}$ on $(0, \infty)$, which has the representation

$$
g_{X_{t}}(r)=\mathbb{E}\left[\frac{1}{r \sigma_{Z}(t)} \phi\left(\frac{\ln \left(r / X_{0}\right)-\mu_{Z}(t)}{\sigma_{Z}(t)}\right)\right]
$$

If

$$
\mathbb{E}\left(\sigma_{Z}^{2}(t)\right)^{-1 / 2}<\infty
$$

then the density function $g_{X_{t}}$ is continuous.

Proof. Notice that we can represent $W$ in the form

$$
W_{t}=\rho Z_{t}+\sqrt{1-\rho^{2}} B_{t},
$$

where $(B, Z)$ is the standard two-dimensional Wiener process. This together with (3) implies that

$$
\ln X_{t}=\ln X_{0}+\theta_{Z}(t)+\theta_{B}(t)
$$


where

$$
\begin{aligned}
\theta_{Z}(t) & :=\rho \int_{0}^{t} Y_{u} d Z_{u}-\frac{1}{2} \rho^{2} \int_{0}^{t} Y_{u}^{2} d u \\
\theta_{B}(t) & :=\sqrt{1-\rho^{2}} \int_{0}^{t} Y_{u} d B_{u}-\frac{1}{2}\left(1-\rho^{2}\right) \int_{0}^{t} Y_{u}^{2} d u .
\end{aligned}
$$

For fixed $r>0$ we have

$$
\begin{aligned}
\mathbb{P}\left(X_{t} \leq r\right) & =\mathbb{E}_{\left\{X_{0} \exp \left(\int_{0}^{t} Y_{u} d W_{u}-\frac{1}{2} \int_{0}^{t} Y_{u}^{2} d u\right) \leq r\right\}} \\
& =\mathbb{E} \mathbb{E}\left[\mathbb{1}_{\left\{\rho \int_{0}^{t} Y_{u} d Z_{u}+\sqrt{1-\rho^{2}} \int_{0}^{t} Y_{u} d B_{u}-\frac{1}{2} \int_{0}^{t} Y_{u}^{2} d u \leq \ln \left(r / X_{0}\right)\right\}} \mid \mathcal{F}_{t}^{Z}\right] .
\end{aligned}
$$

Since SDE (2) has the unique strong solution, there exists an appropriately measurable function $\Psi(\cdot, \cdot)$ such that $Y=\Psi\left(Y_{0}, Z\right)$ (see, e.g. Ikeda, Watanabe [7]). Together with the fact that the processes $B$ and $Z$ are independent Brownian motions, this implies that the random variable $\theta_{B}(t)$, for a fixed trajectory of $Z_{u}, u \leq t$, has the Gaussian distribution with mean $\hat{\mu}=-\frac{1}{2}\left(1-\rho^{2}\right) \int_{0}^{t} Y_{u}^{2} d u$ and variance $\hat{\sigma}^{2}=\left(1-\rho^{2}\right) \int_{0}^{t} Y_{u}^{2} d u$. Consequently, by 11 , we obtain

$$
\begin{aligned}
\mathbb{P}\left(X_{t} \leq r\right) & =\mathbb{E} \mathbb{P}\left(\mu_{Z}(t)+\sigma_{Z}(t) g \leq \ln \frac{r}{X_{0}} \mid \mathcal{F}_{t}^{Z}\right) \\
& =\mathbb{E} \mathbb{P}\left(g \leq \frac{\ln \left(r / X_{0}\right)-\mu_{Z}(t)}{\sigma_{Z}(t)} \mid \mathcal{F}_{t}^{Z}\right)=\mathbb{E} \Phi\left(\frac{\ln \left(r / X_{0}\right)-\mu_{Z}(t)}{\sigma_{Z}(t)}\right),
\end{aligned}
$$

where $g$ is a standard Gaussian random variable independent of $\mathcal{F}_{t}^{Z}, \mu_{Z}(t)$ and $\sigma_{Z}^{2}(t)$ are given by (5) and (6), respectively. This finishes the proof of (4).

Since

$$
\frac{\partial}{\partial r} \Phi\left(\frac{\ln \left(r / X_{0}\right)-\mu_{Z}(t)}{\sigma_{Z}(t)}\right)=\frac{1}{r \sigma_{Z}(t)} \phi\left(\frac{\ln \left(r / X_{0}\right)-\mu_{Z}(t)}{\sigma_{Z}(t)}\right)
$$

we have, by the Fubini theorem for nonnegative functions,

$$
\begin{aligned}
\mathbb{P}\left(X_{t} \leq r\right) & =\mathbb{E} \int_{0}^{r} \frac{1}{s \sigma_{Z}(t)} \phi\left(\frac{\ln \left(s / X_{0}\right)-\mu_{Z}(t)}{\sigma_{Z}(t)}\right) d s \\
& =\int_{0}^{r} \mathbb{E}\left[\frac{1}{s \sigma_{Z}(t)} \phi\left(\frac{\ln \left(s / X_{0}\right)-\mu_{Z}(t)}{\sigma_{Z}(t)}\right)\right] d s
\end{aligned}
$$

for $r>0$. Hence the random variable $X_{t}$ has the density function $g_{X_{t}}$ given by (7).

The continuity of density, under assumption (8), follows from (7) and the Lebesgue dominated convergence theorem. Indeed, fix an arbitrary $r>0$. Observe that

$$
s \longmapsto \frac{1}{s \sigma_{Z}(t)} \phi\left(\frac{\ln \left(s / X_{0}\right)-\mu_{Z}(t)}{\sigma_{Z}(t)}\right)
$$

is continuous on $(0, \infty)$, and

$$
\frac{1}{s \sigma_{Z}(t)} \phi\left(\frac{\ln \left(s / X_{0}\right)-\mu_{Z}(t)}{\sigma_{Z}(t)}\right) \leq \frac{1}{r-\epsilon}\left(\frac{1}{\sigma_{Z}(t)}\right) \phi\left(\frac{\ln \left((r+\epsilon) / X_{0}\right)-\mu_{Z}(t)}{\sigma_{Z}(t)}\right):=J
$$

for $s \in(r-\epsilon, r+\epsilon)$. Since, by (8), $J$ is integrable, we conclude that $\lim _{s \rightarrow r} g_{X_{t}}(s)=g_{X_{t}}(r)$ by the Lebesgue dominated convergence theorem. 
REMARK 3.2. From the last theorem it is clear, under our standing assumption $|\rho|<1$, that finding the distribution of $X_{t}$, for fixed $t$, reduces to deriving the distribution of the vector $\left(\int_{0}^{t} Y_{u} d Z_{u}, \int_{0}^{t} Y_{u}^{2} d u\right)$, so to finding functionals of Brownian motion. For the case $\rho= \pm 1$ we have the same conclusion because in this case we have $W= \pm Z$ and

$$
X_{t}=X_{0} \exp \left( \pm \int_{0}^{t} Y_{u} d Z_{u}-\frac{1}{2} \int_{0}^{t} Y_{u}^{2} d u\right)
$$

In the next proposition we give two sufficient conditions for $(8)$ to hold.

Proposition 3.3. Assume that one of the following conditions holds:

i) There exists $m \geq 1$ such that

$$
\mathbb{E}\left(\int_{0}^{t} Y_{u}^{2} d u\right)^{-m / 2}<\infty
$$

ii) There exists $\beta>0$ and $m \geq \frac{1}{2 \beta}$ such that

$$
\mathbb{E}\left(\int_{0}^{t} Y_{u}^{-2 \beta} d u\right)^{m}<\infty
$$

Then condition (8) is satisfied.

\section{Proof.}

i) Using the Hölder inequality we see that $(13)$ implies $(8)$ for $m \geq 1$.

ii) Assume that 14 holds. Since, by the Hölder inequality,

$$
t \leq\left(\int_{0}^{t} Y_{u}^{2} d u\right)^{\beta /(\beta+1)}\left(\int_{0}^{t} Y_{u}^{-2 \beta} d u\right)^{1 /(1+\beta)},
$$

we have

$$
E\left(\int_{0}^{t} Y_{u}^{2} d u\right)^{-1 / 2} \leq t^{-(\beta+1) /(2 \beta)} E\left(\int_{0}^{t} Y_{u}^{-2 \beta} d u\right)^{1 /(2 \beta)} .
$$

Hence, using again the Hölder inequality with $p=2 m \beta \geq 1$, we conclude that (14) implies (8).

Now, we formulate the conditions ensuring that the density function of $X_{t}$ belongs to the class $C^{\infty}=\mathcal{C}^{\infty}((0, \infty))$ and present an analytic form of the density. An application of this proposition will be presented in Theorem 4.7. In what follows by $f^{(n)}$ we denote the $n$-th derivative of $f$.

Proposition 3.4. Fix $t \in(0, T]$. Let $X_{0}=x>0$. Assume that

$$
\mathbb{E}\left|\frac{1}{\sigma_{Z}^{n+1}(t)}\left(\frac{\mu_{Z}(t)}{\sigma_{Z}(t)}\right)^{p} \phi^{(n)}\left(\frac{\ln (r / x)-\mu_{Z}(t)}{\sigma_{Z}(t)}\right)\right|<\infty
$$

for every $r \in(0, \infty), n \in \mathbb{N}, p \in\{0,1\}$, and

$$
\mathbb{E} \sigma_{Z}^{-m}(t)<\infty
$$

for every $m \in \mathbb{N}$. Then the density function $g_{X_{t}}$ belongs to the class $\mathcal{C}^{\infty}$. Moreover, if

$$
\lim _{n \rightarrow \infty} \frac{r^{n}}{n !} \mathbb{E}\left(\frac{1}{\sigma_{Z}^{n+1}(t)} \phi^{(n)}\left(\frac{r-\mu_{Z}(t)}{\sigma_{Z}(t)}\right)\right)=0,
$$


for every $r \in \mathbb{R}$, then

$$
g_{X_{t}}(r)=\frac{1}{r}\left(\sum_{n=0}^{\infty} \gamma_{n}(\ln (r / x))^{n}\right),
$$

where the coefficients $\gamma_{k}$ (depending on fixed $t$ ) are given by

$$
\gamma_{k}=\mathbb{E}\left(\frac{1}{\sigma_{Z}^{k}(t)} \phi^{(k-1)}\left(-\frac{\mu_{Z}(t)}{\sigma_{Z}(t)}\right)\right) .
$$

Proof. Theorem 3.1 implies, by assumption (16), that $r \mapsto g_{X_{t}}(r)$ is continuous on $(0, \infty)$. Using similar arguments as in the proof of Theorem 3.1. we can differentiate $g$ and we obtain

$$
g_{X_{t}}^{\prime}(r)=\frac{1}{r} \mathbb{E}\left(\frac{1}{\sigma_{Z}^{2}(t)} \phi^{\prime}\left(\frac{\ln (r / x)-\mu_{Z}(t)}{\sigma_{Z}(t)}\right)\right)-\frac{1}{r^{2}} \mathbb{E}\left(\frac{1}{\sigma_{Z}(t)} \phi\left(\frac{\ln (r / x)-\mu_{Z}(t)}{\sigma_{Z}(t)}\right)\right) .
$$

Now, using assumptions (15), (16), the elementary formula

$$
\phi^{(k+1)}(x)=-x \phi^{(k)}(x)-k \phi^{(k-1)}(x)
$$

and proceeding as in the proof of Theorem 3.1 we deduce that the function

$$
r \mapsto \mathbb{E}\left(\frac{1}{\sigma_{Z}^{n+1}(t)} \phi^{(n)}\left(\frac{\ln (r / x)-\mu_{Z}(t)}{\sigma_{Z}(t)}\right)\right)
$$

is differentiable. By similar arguments, using mathematical induction, the fact that

$$
\mathbb{E}\left|\frac{\ln (r / x)-\mu_{Z}(t)}{\sigma_{Z}^{n+2}(t)} \phi^{(n)}\left(\frac{\ln (r / x)-\mu_{Z}(t)}{\sigma_{Z}(t)}\right)\right|<\infty,
$$

and the Leibniz formula we infer the first statement.

To prove the second statement let us define a function $H$ on $\mathbb{R}$ by formula

$$
H(\theta)=\mathbb{E}\left(\frac{1}{\sigma_{Z}(t)} \phi\left(\frac{\theta-\mu_{Z}(t)}{\sigma_{Z}(t)}\right)\right) .
$$

By similar arguments as above we see that $H \in C^{\infty}$. If condition 17 holds, then $H$ can be expanded into the series

$$
H(\theta)=\sum_{n=0}^{\infty} \gamma_{n} \theta^{n}
$$

The sequence $\left(\gamma_{k}\right)$ we find in a standard way by taking derivatives of $H$ at point 0 . By Theorem 3.1, for $\theta=\ln (r / x)$, we have

$$
g_{X_{t}}(r)=\frac{1}{r} H(\ln (r / x)) .
$$

Combining this and $(20)$ we finish the proof.

In the next few theorems we present some properties of $X$.

Theorem 3.5. Assume that $X_{0}=x, \mathbb{E} \int_{0}^{T} X_{u}^{2} Y_{u}^{2} d u<\infty, \mathbb{E} \sigma_{Z}^{2}(t)^{-1}<\infty$. Let $\gamma$ be a gamma random variable with parameter $1 / 2$ independent from $X$ and

$$
G(z):=\frac{1}{4} 1_{\{(0, \infty)\}}(z)\left(\frac{x}{2 z^{2}}-\frac{3}{z}\right) .
$$


Let us define, for $t \geq 0$,

$$
\begin{aligned}
H_{t}(z) & :=\mathbb{E}\left(Y_{t}^{2} \mid X_{t}=z\right), \\
F_{t}(z) & :=\mathbb{E}\left(G(\gamma) H_{t}\left(X_{t}\right) \mid \frac{X_{t}^{2}}{4 \gamma}=z\right) .
\end{aligned}
$$

Assume that, for every $z>0$, the functions $t \mapsto H_{t}, t \mapsto F_{t}$ are continuous. Then

$$
\mathbb{P}\left(\frac{X_{t}^{2}}{4 \gamma} \in d z\right)=\frac{x}{2 \sqrt{\pi z^{3}}} \exp \left(-\frac{x^{2}}{4 z}+\int_{0}^{t} F_{u}(z) d u\right) \mathbb{1}_{(0, \infty)}(z) d z
$$

Proof. Let us recall that $X_{t}=x+\int_{0}^{t} X_{u} Y_{u}\left(\rho d B_{u}+\sqrt{1-\rho^{2}} d V_{u}\right)$, where $B, V$ are two independent Brownian motions. From the Itô lemma we have

$$
d e^{-\lambda X_{t}}=-\lambda e^{-\lambda X_{t}} X_{t} Y_{t}\left(\rho d B_{u}+\sqrt{1-\rho^{2}} d V_{u}\right)+\frac{\lambda^{2}}{2} e^{-\lambda X_{t}} X_{t}^{2} Y_{t}^{2} d t
$$

for $\lambda>0$. The local martingale in 23 is a true martingale since $\int_{0}^{T} \mathbb{E} X_{u}^{2} Y_{u}^{2} d u<\infty$, by assumption, so

$$
\mathbb{E} e^{-\lambda X_{t}}=e^{-\lambda x}+\frac{\lambda^{2}}{2} \mathbb{E} \int_{0}^{t} e^{-\lambda X_{u}} X_{u}^{2} Y_{u}^{2} d u=e^{-\lambda x}+\frac{\lambda^{2}}{2} \mathbb{E} \int_{0}^{t} e^{-\lambda X_{u}} X_{u}^{2} H_{u}\left(X_{u}\right) d u .
$$

Hence the function $t \mapsto \mathbb{E} e^{-\lambda X_{t}}$ is differentiable for $t>0$, and

$$
\frac{\partial}{\partial t} \mathbb{E} e^{-\lambda X_{t}}=\frac{\lambda^{2}}{2} \mathbb{E}\left(e^{-\lambda X_{t}} X_{t}^{2} H_{t}\left(X_{t}\right)\right)
$$

Using the fact that $\mathbb{E} e^{-\lambda^{2} /(4 \gamma)}=e^{-\lambda}$ and independence of $X_{t}$ and $\gamma$ we have

$$
\frac{\lambda^{2}}{2} \mathbb{E}\left(e^{-\lambda X_{t}} X_{t}^{2} H_{t}\left(X_{t}\right)\right)=\frac{1}{2} \mathbb{E}\left(e^{-\lambda^{2} X_{t}^{2} /(4 \gamma)} \lambda^{2} X_{t}^{2} H_{t}\left(X_{t}\right)\right)
$$

The density of $\frac{1}{4 \gamma}$ given by $f(z)=\frac{x}{2 \sqrt{\pi z^{3}}} e^{-x^{2} /(4 z)} \mathbb{1}_{(0, \infty)}(z)$ is differentiable on $(0, \infty)$ and $f(0)=0$. Moreover, $G=\frac{1}{2} \frac{f^{\prime}}{f}$, thus we obtain using integration by parts

$$
\begin{array}{r}
\frac{1}{2} \mathbb{E}\left(e^{-\lambda^{2} X_{t}^{2} /(4 \gamma)} \lambda^{2} X_{t}^{2} H_{t}\left(X_{t}\right)\right)=\frac{1}{2} \int_{0}^{\infty} \int_{0}^{\infty} e^{-\lambda^{2} u^{2} z} \lambda^{2} u^{2} H_{t}(u) f(z) d z \mathbb{P}_{X_{t}}(d u) \\
=\frac{1}{2} \int_{0}^{\infty} \int_{0}^{\infty} e^{-\lambda^{2} u^{2} z} H_{t}(u) f^{\prime}(z) d z \mathbb{P}_{X_{t}}(d u)=\mathbb{E}\left(e^{-\lambda^{2} X_{t}^{2} /(4 \gamma)} G(\gamma) H_{t}\left(X_{t}\right)\right) .
\end{array}
$$

Hence and from 24 and 25 we conclude, for $\xi_{t}:=\frac{X_{t}^{2}}{4 \gamma}$, that

$$
\frac{\partial}{\partial t} \mathbb{E} e^{-\lambda^{2} \xi_{t}}=\mathbb{E}\left(e^{-\lambda^{2} \xi_{t}} G(\gamma) H_{t}\left(X_{t}\right)\right)
$$

Observe that from (7) we have

$$
\mathbb{P}\left(\xi_{t} \in d v\right)=\frac{1}{2 v} \mathbb{E}\left[\frac{1}{\sigma_{Z}(t)} \phi\left(\frac{\ln (2 \sqrt{v \gamma} / x)-\mu_{Z}(t)}{\sigma_{Z}(t)}\right)\right] d v,
$$

so $t \mapsto g_{\xi_{t}}(z) d z:=\mathbb{P}\left(\xi_{t} \in d z\right)$ is differentiable since $\mathbb{E}\left(\sigma_{Z}^{2}(t)\right)^{-1}<\infty$. Next, we conclude from 26 that

$$
\frac{\partial}{\partial t} g_{\xi_{t}}(z)=g_{\xi_{t}}(z) F_{t}(z)
$$


Moreover, $\xi_{0}$ has the density

$$
g_{\xi_{0}}(z) d z=\mathbb{P}\left(\frac{x^{2}}{4 \gamma} \in d z\right)=\frac{x}{2 \sqrt{\pi z^{3}}} e^{-x^{2} /(4 z)} \mathbb{1}_{(0, \infty)}(z) d z .
$$

By assumption, $t \mapsto F_{t}$ and $t \mapsto H_{t}$ are continuous. Thus, using (27) and (28) we conclude that

$$
g_{\xi_{t}}(z)=\frac{x}{2 \sqrt{\pi z^{3}}} \exp \left(-\frac{x^{2}}{4 z}+\int_{0}^{t} F_{u}(z) d u\right) \mathbb{1}_{(0, \infty)}(z),
$$

which is the statement of the theorem.

Hence we deduce an interesting factorization of the density of $\xi_{t}=\frac{X_{t}^{2}}{4 \gamma}$.

COROLlaRY 3.6. Under assumptions of Theorem 3.5, for $z>0$ we have

$$
\mathbb{P}\left(\frac{X_{t}^{2}}{4 \gamma} \in d z\right)=\mathbb{P}\left(\frac{x^{2}}{4 \gamma} \in d z\right) \exp \left(\int_{0}^{t} F_{u}(z) d u\right) .
$$

ii)

$$
\mathbb{E}\left(G(\gamma) H_{t}\left(X_{t}\right) \mid \frac{X_{t}^{2}}{4 \gamma}=z\right)=\frac{\mathbb{E}\left(G(\gamma) H(2 \sqrt{z \gamma}) \sqrt{\gamma} g_{X_{t}}(2 \sqrt{z \gamma})\right)}{\mathbb{E}\left(\sqrt{\gamma} g_{X_{t}}(2 \sqrt{z \gamma})\right)}
$$

Proof.

i) It follows immediately from 22 and 28 .

ii) The assertion follows from the fact that for two positive independent random variables $X$ and $Y$ having densities $g_{X}$ and $g_{Y}$ respectively,

$$
\mathbb{P}\left(Y \in d y \mid \frac{X}{Y}=z\right)=\frac{g_{Y}(y) y g_{X}(y z)}{\mathbb{E}\left(Y g_{X}(Y z)\right)},
$$

for $z>0$.

From Corollary 3.6 it follows that to know the density of $X_{t}^{2} /(4 \gamma)$ it is enough to know the form of function $F$. As we will see in the next proposition the distribution of $X_{t}^{2} /(4 \gamma)$ leads to the explicit formula of modified Laplace transform of $X_{t}^{2}$.

Proposition 3.7. Under the assumptions of Theorem 3.5, for any $v>0$ we have

$$
\mathbb{E}\left(X_{t} \exp \left(-\frac{X_{t}^{2}}{4 v}\right)\right)=x \exp \left(-\frac{x^{2}}{4 v}+\int_{0}^{t} F_{u}(v) d u\right) .
$$

Proof. Let $\xi_{t}=\frac{X_{t}^{2}}{4 \gamma}$. Using subsequently Fubini's theorem, the elementary formula

$$
e^{-\sqrt{\lambda} s}=\frac{s}{\sqrt{2}} \int_{0}^{\infty} \frac{1}{\sqrt{2 \pi v^{3}}} \exp \left(-\lambda v-\frac{s^{2}}{4 v}\right) d v \quad \text { for } s>0
$$

the identity $\mathbb{E} \exp \left(-\frac{\lambda^{2}}{4 \gamma}\right)=e^{-\lambda}$ and Corollary 3.6 we obtain

$$
\begin{aligned}
\mathbb{E} e^{-\lambda^{2} \xi_{t}} & =\mathbb{E} \exp \left(-\lambda^{2} \frac{X_{t}^{2}}{4 \gamma}\right)=\mathbb{E} e^{-\lambda X_{t}}=\mathbb{E}\left(\frac{X_{t}}{\sqrt{2}} \int_{0}^{\infty} \frac{1}{\sqrt{2 \pi v^{3}}} \exp \left(-\lambda^{2} v-\frac{X_{t}^{2}}{4 v}\right) d v\right) \\
& =\int_{0}^{\infty} \frac{1}{2 \sqrt{\pi v^{3}}} e^{-\lambda^{2} v} \mathbb{E}\left(X_{t} \exp \left(-\frac{X_{t}^{2}}{4 v}\right)\right) d v
\end{aligned}
$$


for $\lambda>0$. Hence and from Corollary 3.6 we have

$$
\begin{array}{rl}
\int_{0}^{\infty} \frac{1}{2 \sqrt{\pi v^{3}}} e^{-\lambda^{2} v} & \mathbb{E}\left(X_{t} \exp \left(-\frac{X_{t}^{2}}{4 v}\right)\right) d v \\
= & \mathbb{E} e^{-\lambda^{2} \xi_{t}}=\int_{0}^{\infty} \exp \left(-\lambda^{2} v+\int_{0}^{t} F_{u}(v) d u\right) \frac{x}{2 v \sqrt{\pi v}} \exp \left(-\frac{x^{2}}{4 v}\right) d v
\end{array}
$$

for $\lambda>0$. The assertion follows.

The next theorem states that knowing the moments $\mathbb{E}\left(\ln X_{t}\right)^{n}$ we can compute $\mathbb{E}\left(X_{t} e^{-\lambda X_{t}^{2}}\right)$ under some assumptions and finally the function $F$.

TheOREM 3.8. Fix $\lambda>0, t>0$. Let $c_{n}=\frac{1}{n !} \sum_{k=0}^{\infty} \frac{(-\lambda)^{k}}{k !}(2 k+1)^{n}$. If for every $n \in \mathbb{N}$ we have

$$
\mathbb{E}\left(X_{t} e^{-\lambda X_{t}^{2}}\right)<\infty, \quad \mathbb{E}\left(\ln X_{t}\right)^{n}<\infty
$$

then

$$
\liminf _{n} \mathbb{E}\left(\sum_{l=0}^{n} c_{l}\left(\ln X_{t}\right)^{l}\right)^{+} \geq \mathbb{E} X_{t} e^{-\lambda X_{t}^{2}}
$$

Moreover, if

$$
\lim _{n} \mathbb{E}\left(\sum_{l=0}^{n} c_{l}\left(\ln X_{t}\right)^{l}\right)^{+}=\lim _{n} \mathbb{E} \sum_{l=0}^{n} c_{l}\left(\ln X_{t}\right)^{l} \leq \mathbb{E}\left(X_{t} e^{-\lambda X_{t}^{2}}\right)
$$

then

$$
\mathbb{E}\left(X_{t} e^{-\lambda X_{t}^{2}}\right)=\sum_{l=0}^{\infty} c_{l} \mathbb{E}\left(\ln X_{t}\right)^{l}
$$

Proof. Fix $t \geq 0$ for which 31 is satisfied. As $x^{k}=\sum_{i=0}^{\infty} \frac{(k \ln x)^{i}}{i !}$ and $x e^{-\lambda x^{2}}=$ $\sum_{k=0}^{\infty} \frac{(-\lambda)^{k}}{k !} x^{2 k+1}$ we have

$$
x e^{-\lambda x^{2}}=\sum_{k=0}^{\infty} \frac{(-\lambda)^{k}}{k !} \sum_{i=0}^{\infty} \frac{((2 k+1) \ln x)^{i}}{i !}=\sum_{i=0}^{\infty} c_{i}(\ln x)^{i}
$$

and

$$
\mathbb{E}\left(X_{t} e^{-\lambda X_{t}^{2}}\right)=\mathbb{E} \sum_{n=0}^{\infty} c_{n}\left(\ln X_{t}\right)^{n}
$$

for $c_{n}=\frac{1}{n !} \sum_{k=0}^{\infty} \frac{(-\lambda)^{k}}{k !}(2 k+1)^{n}$. Let $X^{(i)}(t)=c_{i}\left(\ln X_{t}\right)^{i}, i \in \mathbb{N}$. By assumptions $X^{(i)}(t)$ is integrable. For $k \in \mathbb{N}$ let

$$
Z_{n}=\sum_{i=0}^{n} X^{(i)}(t), \quad \Lambda_{k}=\left\{\omega: \sup _{n} Z_{n}(\omega)=Z_{k}(\omega)\right\}, \quad \Lambda=\bigcup_{k=1}^{\infty} \Lambda_{k} .
$$

Integrability of $X_{t}$ yields

$$
\sum_{k=1}^{\infty} \mathbb{E} X_{t} e^{-\lambda X_{t}^{2}} \mathbb{1}_{\Lambda_{k}}=\mathbb{E} X_{t} e^{-\lambda X_{t}^{2}} \mathbb{1}_{\Lambda}
$$


Observe that on $\Lambda^{c}$ we have $\sup _{n} Z_{n}=\sum_{i=0}^{\infty} X^{(i)}(t)$ and the equality

$$
\mathbb{E}\left(X_{t} e^{-\lambda X_{t}^{2}} \mathbb{1}_{\Lambda^{c}}\right)=\sum_{n=0}^{\infty} \mathbb{E}\left(c_{n}\left(\ln X_{t}\right)^{n} \mathbb{1}_{\Lambda^{c}}\right)
$$

follows from the monotone convergence theorem. Define

$$
B_{n}=\left\{Z_{n}(\omega) \geq 0\right\}, \quad \xi_{n}=\sup _{j} Z_{j}-Z_{n} \geq 0 .
$$

From Fatou's lemma, for fixed $k$, we have

$$
\liminf _{n} \mathbb{E} \xi_{n} \mathbb{1}_{\Lambda_{k} \cap B_{n}} \geq \mathbb{E}\left(Z_{k} \mathbb{1}_{\Lambda_{k}}\right)-\mathbb{E}\left(X_{t} e^{-\lambda X_{t}^{2}} \mathbb{1}_{\Lambda_{k}}\right) .
$$

On the other side, the Lebesgue dominated convergence theorem yields

$$
\lim _{n} \mathbb{E} \xi_{n} \mathbb{1}_{\Lambda_{k} \cap B_{n}}=\mathbb{E} Z_{k} \mathbb{1}_{\Lambda_{k}}-\lim _{n} \mathbb{E} Z_{n} \mathbb{1}_{\Lambda_{k} \cap B_{n}},
$$

and thus

$$
\mathbb{E} X_{t} e^{-\lambda X_{t}^{2}} \mathbb{1}_{\Lambda_{k}} \leq \liminf _{n} \mathbb{E} Z_{n} \mathbb{1}_{\Lambda_{k} \cap B_{n}} \leq \mathbb{E} X_{t} e^{-\lambda X_{t}^{2}} \mathbb{1}_{\Lambda_{k}}
$$

where in the first inequality we used again Fatou's lemma. In result for any $k_{1}, k_{2}, \ldots, k_{N}$ and $N \in \mathbb{N}$

$$
\sum_{j=1}^{N} \mathbb{E} X_{t} e^{-\lambda X_{t}^{2}} \mathbb{1}_{\Lambda_{k_{j}}}=\liminf _{n} \mathbb{E} Z_{n} \mathbb{1}_{B_{n}} \mathbb{1}_{\Lambda_{k_{j}}}
$$

Hence, from (35), Fatou's lemma, and Fubini's theorem we obtain

$$
\mathbb{E} X_{t} e^{-\lambda X_{t}^{2}} \mathbb{1}_{\Lambda}=\sum_{k=1}^{\infty} \liminf _{n} \mathbb{E} Z_{n} \mathbb{1}_{\Lambda_{k} \cap B_{n}} \leq \liminf _{n} \sum_{k=1}^{\infty} \mathbb{E} Z_{n} \mathbb{1}_{\Lambda_{k} \cap B_{n}}=\liminf _{n} \mathbb{E}\left(Z_{n}^{+} \mathbb{1}_{\Lambda}\right) .
$$

If we assume 33 , then

$$
\liminf _{n} \mathbb{E} Z_{n}^{+} \leq \lim _{n} \mathbb{E} Z_{n}^{+}=\lim _{n} \mathbb{E} Z_{n} \leq \mathbb{E}\left(X_{t} e^{-\lambda X_{t}^{2}}\right)
$$

and the assertion follows from 32 .

In the next section we describe a recursive procedure for calculation of $\mathbb{E}\left(\ln X_{t}\right)^{n}$ in the lognormal stochastic volatility model.

4. Log-normal stochastic volatility model. In this section we present how our general results can be used for the specific models. Using our general framework we obtain the refined results for the log-normal stochastic volatility model.

A log-normal model is a model where the process $Y$ is a geometric Brownian motion, so

$$
Y_{t}=y e^{\sigma Z_{t}-t \sigma^{2} / 2}, \quad y>0, \quad \sigma>0 .
$$

It was considered by Hull and White in the case of uncorrelated noises [6]. In the case of correlated noises it is a SABR model with $\beta=1$, introduced in 2002 by Hagan et al. [5]. Sin [16] and later Jourdain [10] proved that in the log-normal stochastic volatility model the price process $X$ is a martingale if and only if $\rho \leq 0$. Their rather technically complicated proof relied on Feller's test for explosion. Another proof can be found in Lions and Musiela [12]. A simple proof of this result can be found in [8]. 
From now, for simplicity of presentation, we assume that $Y_{t}=y e^{Z_{t}-t / 2}$. The results below can be deduced with some effort for the process $y e^{\sigma Z_{t}-t \sigma^{2} / 2}, \sigma>0$, by the scaling of time. The first result is an application of the representation of density given by (7).

TheOrem 4.1. Fix $t>0$. Let $Y_{t}=y e^{Z_{t}-t / 2}$ and $X_{0}=x>0$. Then

$$
\begin{aligned}
g_{X_{t}}(r) & =\frac{2 e^{-t / 8}}{r y \sqrt{2 \pi\left(1-\rho^{2}\right)}} \frac{1}{h(x, r, y, \rho)} \\
& \times \int_{0}^{\infty} H(z, y, \rho) K_{-1}(H(z, y, \rho) h(x, r, y, \rho)) e^{q(x, r, y, \rho, z)} \frac{\theta(z, t)}{z^{3 / 2}} d z
\end{aligned}
$$

where $K_{-1}$ is the modified Bessel function and

$$
\begin{aligned}
h(x, r, y, \rho) & =\frac{1}{y \sqrt{1-\rho^{2}}} \sqrt{y^{2}\left(1-\rho^{2}\right)+(\ln (r / x)+\rho y)^{2}}, \\
\theta(z, t) & =\frac{z}{\sqrt{2 \pi^{3} t} e^{\pi^{2} /(2 t)} \int_{0}^{\infty} e^{-u^{2} /(2 t)} e^{-z \cosh (u)} \sinh (u) \sin (\pi u / t) d u} \\
H(z, y, \rho) & =\frac{1}{\sqrt{1-\rho^{2}}} \sqrt{z^{2}\left(1-\rho^{2}\right)+(\rho z-y / 2)^{2}}, \\
q(x, r, y, \rho, z) & =\frac{1}{2 y\left(1-\rho^{2}\right)}(\ln (r / x)+\rho y)(2 \rho z-y)
\end{aligned}
$$

for $z \geq 0$.

Proof. Let $T_{\lambda}$ be an exponential random variable with parameter $\lambda$, independent of $\mathbb{F}^{Y, X}$. From (7) we have

$$
\mathbb{E} g_{X_{T_{\lambda}}}(r)=\mathbb{E}\left[\frac{1}{r \sigma_{Z}\left(T_{\lambda}\right)} \phi\left(\frac{\ln (r / x)-\mu_{Z}\left(T_{\lambda}\right)}{\sigma_{Z}\left(T_{\lambda}\right)}\right)\right],
$$

where $\mu_{Z}(t)=\rho\left(Y_{t}-y\right)-\frac{1}{2} A_{t}, \sigma_{Z}(t)=\sqrt{1-\rho^{2}} \sqrt{A_{t}}, A_{t}=\int_{0}^{t} Y_{u}^{2} d u$. Hence and from Theorem 4.11 Matsumoto and Yor [13] describing the joint density of the vector $\left(e^{B_{T_{\lambda}}-T_{\lambda} / 2}, \int_{0}^{T_{\lambda}} e^{2 B_{u}-u} d u\right)$ we conclude that

$$
\begin{array}{r}
\mathbb{E} g_{X_{T_{\lambda}}}(r)=\frac{1}{r y \sqrt{2 \pi\left(1-\rho^{2}\right)}} \int_{0}^{\infty} \int_{0}^{\infty} \frac{1}{\sqrt{u}} \exp \left(-\frac{\left(\ln (r / x)-\rho y(v-1)+y^{2} u / 2\right)^{2}}{2\left(1-\rho^{2}\right) y^{2} u}\right) \\
\times \frac{\lambda}{v^{2+\gamma+1 / 2}} p^{(\gamma)}(u, 1, v) d u d v
\end{array}
$$

where $p^{(\gamma)}$ is the transition probability density of the Bessel process with index $\gamma$, and here $\gamma=\sqrt{2 \lambda+1 / 4}$. Let us substitute $v=z u$. The last expression is equal to

$$
\frac{\lambda}{r y \sqrt{2 \pi\left(1-\rho^{2}\right)}} \int_{0}^{\infty} z^{-3 / 2} I_{\gamma}(z) e^{q(x, r, y, \rho, z)} \int_{0}^{\infty} \frac{1}{u^{2}} e^{-\left(h^{2}(x, r, y, \rho) u^{-1}+H^{2}(z, y, \rho) u\right) / 2} d u d z,
$$

where $I_{\gamma}$ is the modified Bessel function. Identifying the GIG density in the last expression we see further that this equals

$$
\frac{\lambda}{r y \sqrt{2 \pi\left(1-\rho^{2}\right)}} \int_{0}^{\infty} z^{-3 / 2} I_{\gamma}(z) e^{q(x, r, y, \rho, z)} 2 K_{-1}(H(z, y, \rho) h(x, r, y, \rho)) \frac{H(x, r, y, \rho)}{h(z, y, \rho)} d z .
$$


To finish the proof we use another identity for modified Bessel functions

$$
I_{\gamma}(z)=\int_{0}^{\infty} e^{-\gamma^{2} t / 2} \theta(z, t) d t, \quad z>0
$$

(see [13, (2.10)]), Fubini's theorem and the standard Laplace transform argument.

REMARK 4.2. Formula (38) giving density of $X_{t}$ is the improvement of the formula presented in Theorem 4.15 of [4] which consists of 3 complicated integrals. In this point we have to mention that $\theta(z, t)$ giving the Hartman-Watson distribution (see, e.g., 13, Theorem A.1]) is an oscillating function for small $t$. So, is not easy to handle with by using numerical methods, see Barrieu, Rouault and Yor [1]. Some methodology of approximating $\theta(z, t)$ nearby 0 useful for numerical applications was presented in Gerhold [3].

Continuing the argumentation from Theorem 4.1 we can find another form of integral in 38 , so we can present a result which gives another form of density of $X_{t}$.

TheOrem 4.3. Fix $t>0$ and $X_{0}=x>0$. The density function of $X_{t}$ in the lognormal stochastic volatility model is given by

$$
\begin{aligned}
g_{X_{t}}(r) & =\frac{2 e^{-t / 8}}{r y \sqrt{2 \pi\left(1-\rho^{2}\right)}} \frac{\exp \left(-\frac{\ln (r / x)+\rho y}{2\left(1-\rho^{2}\right)}\right)}{h^{2}(x, r, y, \rho)} \\
& \times \int_{0}^{\infty} \exp \left(-s-\frac{y^{2} h^{2}(x, r, y, \rho)}{16 s\left(1-\rho^{2}\right)}\right) w\left(\alpha_{x, r, y, \rho}(s), \beta_{x, r, y, \rho}(s)\right) d s,
\end{aligned}
$$

where

$$
w(\alpha, \beta)=\int_{0}^{\infty} z^{-3 / 2} e^{-\alpha z^{2}+\beta z} \theta(z, t) d z, \quad \alpha>0, \beta \in \mathbb{R},
$$

and for $s>0$

$$
\alpha_{x, r, y, \rho}(s)=\frac{h^{2}(x, r, y, \rho)}{4 s\left(1-\rho^{2}\right)}, \quad \beta_{x, r, y, \rho}(s)=\frac{\rho}{1-\rho^{2}}\left(\frac{y}{4 s} h^{2}(x, r, y, \rho)+\frac{\ln (r / x)+\rho y}{y}\right)
$$

with $h$ and $\theta$ given by $(39$ and 40 .

Proof. Using the integral representation of modified Bessel function $K_{-1}$

$$
K_{-1}(u)=\frac{1}{u} \int_{0}^{\infty} e^{-s-u^{2} /(4 s)} d s
$$

(see Lebedev [11, p. 119]), and the Fubini theorem we obtain

$$
\begin{aligned}
& \frac{1}{h(x, r, y, \rho)} \int_{0}^{\infty} H(z, y, \rho) K_{-1}(H(z, y, \rho) h(x, r, y, \rho)) e^{q(x, r, y, \rho, z)} \frac{\theta(z, t)}{z^{3 / 2}} d z \\
= & \int_{0}^{\infty} \int_{0}^{\infty} \frac{1}{h^{2}(x, r, y, \rho)} \exp \left(-s-\frac{H^{2}(z, y, \rho) h^{2}(x, r, y, \rho)}{4 s}\right) e^{q(x, r, y, \rho, z)} \frac{\theta(z, t)}{z^{3 / 2}} d z d s \\
= & \frac{e^{-(\ln (r / x)+\rho y) /\left(2\left(1-\rho^{2}\right)\right)}}{h^{2}(x, r, y, \rho)} \int_{0}^{\infty} \exp \left(-s-\frac{y^{2} h^{2}(x, r, y, \rho)}{16 s\left(1-\rho^{2}\right)}\right) w\left(\alpha_{x, r, y, \rho}(s), \beta_{x, r, y, \rho}(s)\right) d s .
\end{aligned}
$$

This and 38 give 42 . 
Proposition 4.4. Fix $t, \rho, y=Y_{0}$. Assume that one of the following conditions holds:

i) $\lambda=0$,

ii) $0<\lambda<1 / y$ and $\rho^{2}>\frac{-y(\lambda+1)}{y \lambda-1}$,

iii) $\lambda \notin[0,1 / y]$ and $\rho^{2}<\frac{-y(\lambda+1)}{y \lambda-1}$.

Then

$$
\begin{gathered}
\mathbb{E} e^{-\lambda \rho y}\left(y^{2}\left(1-\rho^{2}\right)\left(\frac{X_{t}}{x}\right)^{-\lambda}+\left(\ln \left(X_{t} / x\right)+\rho y\right)^{2}\left(\frac{X_{t}}{x}\right)^{-\lambda}\right) \\
=\sqrt{\pi} y^{2} e^{-t / 8}\left(1-\rho^{2}\right) \int_{0}^{\infty} \frac{\theta(z, t)}{z^{3 / 2} \sqrt{z^{2}+\zeta^{2}(z, y, \rho)}}(\psi(z, y, \rho, \lambda))^{-3 / 2} \\
\times \exp \left(-\sqrt{\left(z^{2}+\zeta^{2}(z, y, \rho)\right) \psi(z, y, \rho, \lambda)}\right)\left(1+\sqrt{\left(z^{2}+\zeta^{2}(z, y, \rho)\right) \psi(z, y, \rho, \lambda)}\right) d z,
\end{gathered}
$$

where

$$
\begin{aligned}
\zeta^{2}(z, y, \rho) & =\left(z \rho-\frac{y}{2}\right)^{2}\left(1-\rho^{2}\right)^{-1} \\
\psi(z, y, \rho, \lambda) & =1-y^{2} \frac{1-\rho^{2}}{z^{2}+\zeta^{2}(z, y, \rho)}\left(\frac{z \rho-(y / 2)}{1-\rho^{2}}-\lambda\right)^{2} .
\end{aligned}
$$

Proof. Using Theorem 4.1 we have

$$
\begin{aligned}
& \mathbb{E}\left(\left(y^{2}\left(1-\rho^{2}\right)+\left(\ln \left(X_{t} / x\right)+\rho y\right)^{2}\right)\left(\frac{X_{t}}{x}\right)^{-\lambda} e^{-\lambda \rho y}\right) \\
& =\int_{0}^{\infty}\left(y^{2}\left(1-\rho^{2}\right)+(\ln (r / x)+\rho y)^{2}\right)\left(\frac{r}{x}\right)^{-\lambda} e^{-\lambda \rho y} \frac{2 e^{-t / 8}}{r y \sqrt{2 \pi\left(1-\rho^{2}\right)}} \frac{1}{h(x, r, y, \rho)} \\
& \quad \times\left(\int_{0}^{\infty} H(z, y, \rho) K_{-1}(H(z, y, \rho) h(x, r, y, \rho)) e^{q(x, r, y, \rho, z)} \frac{\theta(z, t)}{z^{3 / 2}} d z\right) d r .
\end{aligned}
$$

Next, using substitution $u=\ln (r / x)+\rho y$, the Fubini theorem, formula (44), the form of functions $h$ and $H$ we see that the last expression is equal to

$$
\begin{aligned}
\int_{0}^{\infty} \int_{0}^{\infty} & 2 y \sqrt{\frac{1-\rho^{2}}{2 \pi}} e^{-t / 8} \frac{\theta(z, t)}{z^{3 / 2}} \exp \left(-s-\frac{1}{4 s}\left(z^{2}+\frac{(z \rho-y / 2)^{2}}{\left(1-\rho^{2}\right)}\right)\right) \\
& \times \int_{-\infty}^{\infty} \exp \left(-\frac{1}{4 s} \frac{u^{2}}{y^{2}\left(1-\rho^{2}\right)}\left(z^{2}+\frac{(z \rho-y / 2)^{2}}{\left(1-\rho^{2}\right)}\right)+u\left(\frac{z \rho-y / 2}{y\left(1-\rho^{2}\right)}-\lambda\right)\right) d u d s d z \\
= & \int_{0}^{\infty} \int_{0}^{\infty} 2 y \sqrt{1-\rho^{2}} e^{-t / 8} \frac{\theta(z, t)}{z^{3 / 2}} \exp \left(-s-\frac{1}{4 s}\left(z^{2}+\frac{(z \rho-y / 2)^{2}}{\left(1-\rho^{2}\right)}\right)\right) \\
& \times y \frac{\sqrt{2 s\left(1-\rho^{2}\right)}}{\sqrt{z^{2}+\frac{(z \rho-y / 2)^{2}}{\left(1-\rho^{2}\right)}}} \exp \left(s y^{2}\left(1-\rho^{2}\right) \frac{\left(\frac{z \rho-y / 2}{\left.z^{2}+\rho^{2}\right)}-\lambda\right)^{2}}{z^{2}+\frac{(z \rho-2)^{2}}{\left(1-\rho^{2}\right)}}\right) d s d z .
\end{aligned}
$$

Now, in the last expression we can again use the form of GIG distribution, since from assumption it follows that

$$
1-y^{2}\left(1-\rho^{2}\right) \frac{\left(\frac{z \rho-y / 2}{y\left(1-\rho^{2}\right)}-\lambda\right)^{2}}{z^{2}+\frac{(z \rho-y / 2)^{2}}{\left(1-\rho^{2}\right)}}>0
$$


for all $z \geq 0$. In result the last integral equals

$$
\begin{aligned}
& \int_{0}^{\infty} 2 y^{2} e^{-t / 8}\left(1-\rho^{2}\right) \frac{\theta(z, t)}{z^{3 / 2}\left(z^{2}+\zeta^{2}(z, y, \rho)\right)^{1 / 2}} \frac{1}{\sqrt{2}} \\
& \quad \times K_{3 / 2}\left(\sqrt{z^{2}+\zeta^{2}(z, y, \rho)} \sqrt{\psi(z, y, \rho, \lambda)}\right)\left(\frac{z^{2}+\zeta^{2}(z, y, \rho)}{\psi(z, y, \rho, \lambda)}\right)^{3 / 4} d z
\end{aligned}
$$

which, due to the fact that $K_{3 / 2}(z)=\frac{\sqrt{\pi}}{\sqrt{2} z^{3 / 2}} e^{-z}(1+z)$, is equal to

$$
\begin{aligned}
& \sqrt{\pi} y^{2} e^{-t / 8}\left(1-\rho^{2}\right) \int_{0}^{\infty} \frac{\theta(z, t)}{z^{3 / 2} \sqrt{z^{2}+\zeta^{2}(z, y, \rho)}}(\psi(z, y, \rho, \lambda))^{-3 / 2} \\
& \quad \times \exp \left(-\sqrt{\left(z^{2}+\zeta^{2}(z, y, \rho)\right) \psi(z, y, \rho, \lambda)}\right)\left(1+\sqrt{\left(z^{2}+\zeta^{2}(z, y, \rho)\right) \psi(z, y, \rho, \lambda)}\right) d z .
\end{aligned}
$$

This finishes the proof.

As an application of this result we obtain a new result for the Hartman-Watson distribution (compare with Remark 4.2.

Corollary 4.5. Let $\rho=0$ and $y=1$. For any $t>0$ and $\lambda \in(-1,0]$

$$
\mathbb{E}\left[\left(1+\ln ^{2}\left(\frac{X_{t}}{x}\right)\right)\left(\frac{X_{t}}{x}\right)^{-\lambda}\right]=\sqrt{\pi} e^{-t / 8} \int_{0}^{\infty} \frac{\theta(z, t)\left(z^{2}+1 / 4\right)}{z^{3 / 2}} \frac{e^{-p(z, \lambda)}}{p^{3}(z, \lambda)}(p(z, \lambda)+1) d z,
$$

where

$$
p(z, \lambda)=\left(z^{2}+\frac{1}{4}-(1 / 2+\lambda)^{2}\right)^{1 / 2} .
$$

Proof. The result follows directly from the last proposition.

Corollary 4.6. For $\rho=0, y=1, t \geq 0$ and $\alpha \in(0,1 / 2)$

$$
\mathbb{E}\left[\left(1+\ln ^{2}\left(\frac{X_{t}}{x}\right)\right)\left(\frac{X_{t}}{x}\right)^{\alpha}\right]=\mathbb{E}\left[\left(1+\ln ^{2}\left(\frac{X_{t}}{x}\right)\right)\left(\frac{X_{t}}{x}\right)^{1-\alpha}\right] .
$$

Proof. We put $\alpha=-\lambda$ and observe that $p(z,-\alpha)=p(z,-(1-\alpha))$ for every $\alpha \in(0,1 / 2)$. The assertion follows from the previous corollary.

As we found the density has complicated form, so it is difficult to calculate density even numerically. Therefore, we present an application of Proposition 3.4 which gives the density of asset price in a linear stochastic volatility model in the form of series 18 with coefficients $\gamma_{k}, k=1, \ldots$, defined by $(19)$. To use Proposition 3.4 we need to verify its assumptions. For this we observe that for $Y_{t}=y e^{Z_{t}-t / 2}$ we have $\mathbb{E}\left(\sigma_{Z}(t)\right)^{a}<\infty$ for any $a \in \mathbb{R}$. The last observation and some tedious algebra lead to the desired conclusion. We omit technical details. To find $\gamma_{k}$ it is enough to compute $\mathbb{E}\left[\frac{\left(\mu_{Z}(t)\right)^{i}}{\left(\sigma_{Z}(t)\right)^{i+j}} \exp \left(-\frac{\left(\mu_{Z}(t)\right)^{2}}{2\left(\sigma_{Z}(t)\right)^{2}}\right)\right]$ for natural $i, j$ since $\gamma_{k}$ is a linear combination of such expressions. 
Proposition 4.7. Let $Y_{t}=y e^{Z_{t}-t / 2}, X_{0}=x>0$ and $i, j \in \mathbb{N}$. Then

$$
\begin{aligned}
& \mathbb{E}\left[\frac{\left(\mu_{Z}(t)\right)^{i}}{\left(\sigma_{Z}(t)\right)^{i+j}} \exp \left(-\frac{\left(\mu_{Z}(t)\right)^{2}}{2\left(\sigma_{Z}(t)\right)^{2}}\right)\right] \\
& =\sum_{k=0}^{i}\left(\begin{array}{l}
i \\
k
\end{array}\right)(-\rho y)^{i-k}\left(1-\rho^{2}\right)^{-(i+j) / 2} h(\rho, y)^{k-(i+j+1) / 2} \exp \left(-\frac{\rho y}{2\left(1-\rho^{2}\right)}-\frac{t}{8}\right) \\
& \times \int_{0}^{\infty}\left(\rho y z-\frac{1}{2}\right)^{k} l(\rho, y, z)^{(i+j+1) / 2-k} 2 K_{k-(i+j+1) / 2}(h(\rho, y) l(\rho, y, z)) \exp \left(\frac{\rho^{2} y^{2} z}{1-\rho^{2}}\right) \theta(z, t) d z
\end{aligned}
$$

where $K_{i}$ are the modified Bessel functions, $\theta$ is given by 40 and

$$
h(\rho, y)=\sqrt{1+\frac{\rho^{2} y^{2}}{1-\rho^{2}}}, \quad l(\rho, y, z)=\frac{1}{2 \sqrt{1-\rho^{2}}} \sqrt{4 z^{2}\left(1-\rho^{2}\right)+(2 \rho y z+1)^{2}} .
$$

Proof. In the proof we use a similar idea as in the proof of Theorem 4.1. We take an exponential random variable $T_{\lambda}$ with parameter $\lambda$, independent of $\mathbb{F}$ and use the form of the joint density of the vector $\left(e^{B_{T_{\lambda}}-T_{\lambda} / 2}, \int_{0}^{T_{\lambda}} e^{2 B_{u}-u} d u\right)$. As a result, we have

$$
\begin{aligned}
& I:=\mathbb{E}\left[\frac{\left(\mu_{Z}\left(T_{\lambda}\right)\right)^{i}}{\left(\sigma_{Z}\left(T_{\lambda}\right)\right)^{i+j}} \exp \left(-\frac{\left(\mu_{Z}\left(T_{\lambda}\right)\right)^{2}}{2\left(\sigma_{Z}\left(T_{\lambda}\right)\right)^{2}}\right)\right] \\
& =\int_{0}^{\infty} \int_{0}^{\infty} \frac{(\rho y(v-1)-u / 2)^{i}}{\left[\left(1-\rho^{2}\right) u y^{2}\right]^{(i+j) / 2}} \exp \left(-\frac{(\rho y(v-1)-u / 2)^{2}}{2 u\left(1-\rho^{2}\right)}\right) \frac{\lambda}{v^{2+\gamma+1 / 2}} p^{(\gamma)}(u, 1, v) d u d v
\end{aligned}
$$

where $p^{(\gamma)}$ is the transition probability density of the Bessel process with index $\gamma=$ $\sqrt{2 \lambda+1 / 4}$. We can rewrite the last expression, using substitution $v=u z$, as

$$
\begin{aligned}
\int_{0}^{\infty} \int_{0}^{\infty} \frac{(\rho y(u z-1)-u / 2)^{i}}{\left[\left(1-\rho^{2}\right) u y^{2}\right]^{(i+j) / 2}} \exp ( & \left.-\frac{1}{2 u} h^{2}(\rho, y)-\frac{u}{2} l^{2}(\rho, y, z)\right) \\
& \times \exp \left(\frac{\rho^{2} y^{2} z}{1-\rho^{2}}-\frac{\rho y}{2\left(1-\rho^{2}\right)}\right) \frac{\lambda}{z^{3 / 2}} \frac{1}{u^{3 / 2}} I_{\gamma}(z) d u d z
\end{aligned}
$$

where $I_{\gamma}$ is the modified Bessel function. Using the Newton formula we expand the expression $(\rho y(u z-1)-u / 2)^{i}$ into the sum and obtain

$$
\begin{aligned}
& I=\sum_{k=0}^{i} \int_{0}^{\infty}\left(\begin{array}{l}
i \\
k
\end{array}\right)\left(\rho y z-\frac{1}{2}\right)^{k}(-\rho y)^{i-k}\left(1-\rho^{2}\right)^{-(i+j) / 2} y^{-(i+j)} \\
& \times\left[\int_{0}^{\infty} u^{k-(i+j+1) / 2-1} \exp \left(-\frac{u}{2} l^{2}(\rho, y, z)-\frac{1}{2 u} h^{2}(\rho, y)\right) d u\right] \\
& \times \exp \left(\frac{\rho^{2} y^{2} z}{1-\rho^{2}}-\frac{\rho y}{2\left(1-\rho^{2}\right)}\right) \frac{\lambda}{z^{3 / 2}} I_{\gamma}(z) d z .
\end{aligned}
$$

Using the GIG distribution we can compute the internal integral and obtain

$$
\begin{aligned}
I=\sum_{k=0}^{i} \int_{0}^{\infty} & \left(\begin{array}{l}
i \\
k
\end{array}\right)\left(\rho y z-\frac{1}{2}\right)^{k}(-\rho y)^{i-k}\left(1-\rho^{2}\right)^{-(i+j) / 2} y^{-(i+j)}\left(\frac{l(\rho, y, z)}{h(\rho, y)}\right)^{-k+(i+j+1) / 2} \\
& \times 2 K_{k-(i+j+1) / 2}(h(\rho, y) l(\rho, y, z)) \exp \left(\frac{\rho^{2} y^{2} z}{1-\rho^{2}}-\frac{\rho y}{2\left(1-\rho^{2}\right)}\right) \frac{\lambda}{z^{3 / 2}} I_{\gamma}(z) d z
\end{aligned}
$$


To finish the proof we again use (41), Fubini's theorem and the standard Laplace transform argument.

In the next proposition we find a recurrence which connects $\mathbb{E}\left(\left(\ln X_{t}\right)^{n} Y_{t}^{k}\right)$ for different values $n \in \mathbb{N}$ and $k \in \mathbb{N} \cup\{0\}$, in particular using this we can compute $\mathbb{E}\left(\ln X_{t}\right)^{n}$ for every $n=1,2, \ldots$

Proposition 4.8. Let $u_{n, k}(t)=\mathbb{E}\left(\left(\ln X_{t}\right)^{n} Y_{t}^{k}\right)$ for $t \geq 0, n \in \mathbb{N}, k \in \mathbb{N} \cup\{0\}$. The functions $u_{n, k}$ satisfy

$$
\begin{aligned}
u_{n, k}^{\prime}(t)=\frac{n(n-1)}{2} u_{n-2, k+2}(t)-\frac{n}{2} u_{n-1, k+2}(t) & \\
& +\frac{k(k-1)}{2} u_{n, k}(t)+n k \rho u_{n-1, k+1}(t) .
\end{aligned}
$$

Proof. By (3), (37), (9) and (10) we see that $u_{n, k}(t)$ are well defined. Moreover,

$$
\mathbb{E} \int_{0}^{t}\left(\ln X_{u}\right)^{2 n} Y_{u}^{2 k} d u<\infty,
$$

so we conclude from Itô's lemma that $u_{n, k} \in \mathcal{C}^{1}$ and 45 holds.

REMARK 4.9. Proposition 4.8 allows us to find values of $u_{n, k}$ in a recursive way. In particular, we can find $u_{n, 0}(t)=\mathbb{E}\left(\ln X_{t}\right)^{n}$. Let us observe that $u_{0, k}(t)=y^{k} e^{t k(k-1) / 2}$, $k \in \mathbb{N} \cup\{0\}$. Moreover, from 45 we have

$$
u_{1, k}^{\prime}(t)=-\frac{1}{2} u_{0, k+2}(t)+\frac{k(k-1)}{2} u_{1, k}(t)+k \rho u_{0, k+1}(t)
$$

for every $k$. From this ODE we obtain $u_{1, k}$ for $k \in \mathbb{N} \cup\{0\}$. To obtain $u_{2, k}$ for $k \in \mathbb{N} \cup\{0\}$ we have to solve the system of ODEs 45 for $n=2$ and $k \in \mathbb{N} \cup\{0\}$, i.e.

$$
u_{2, k}^{\prime}(t)=u_{0, k+2}(t)-u_{1, k+2}(t)+\frac{k(k-1)}{2} u_{2, k}(t)+2 k \rho u_{1, k+1}(t) .
$$

Generally, having calculated $u_{m, k}$ for all $m<n$ and $k \in \mathbb{N} \cup\{0\}$ we find $u_{n, k}$ for all $k \in \mathbb{N} \cup\{0\}$ using 45 . The procedure is completed.

In particular, knowing $u_{n-2,2}$ and $u_{n-1,2}$ we find $\mathbb{E}\left(\ln X_{t}\right)^{n}=u_{n, 0}(t)$ since $u_{n, 0}^{\prime}=$ $\frac{n(n-1)}{2} u_{n-2,2}-\frac{n}{2} u_{n-1,2}$.

\section{References}

[1] P. Barrieu, A. Rouault, M. Yor, A study of the Hartman-Watson distribution motivated by numerical problems related to the pricing of Asian options, J. Appl. Probab. 41 (2004), 1049-1058.

[2] R. Carmona, V. Durrleman, Pricing and hedging spread options, SIAM Rev. 45 (2003), $627-685$.

[3] S. Gerhold, The Hartman-Watson distribution revisited: asymptotics for pricing Asian options, J. Appl. Probab. 48 (2011), 892-899.

[4] A. Gulisashvili, Analytically Tractable Stochastic Stock Price Models, Springer Finance, Springer, Heidelberg, 2012. 
[5] P. Hagan, D. Kumar, A. Lesniewski, D. Woodward, Managing smile risk, Wilmott Magazine, September 2002, 84-108.

[6] J. Hull, A. White, The pricing of options on assets with stochastic volatilities, J. Finance 42 (1987), 281-300.

[7] N. Ikeda, S. Watanabe, Stochastic Differential Equations and Diffusion Processes, NorthHolland, Amsterdam, 1981.

[8] J. Jakubowski, M. Wiśniewolski, A simple proof of the martingale property in a semi-lognormal stochastic volatility model, Appl. Math. (Warsaw) 45 (2018), 1-4.

[9] J. Jakubowski, M. Wiśniewolski, Another look at the Hartman-Watson distributions, Potential Anal. 53 (2020), 1269-1297.

[10] B. Jourdain, Loss of martingality in asset price model with log-normal stochastic volatility, ENPC-CERMICS, Working paper, 2004.

[11] N. N. Lebedev, Special Functions and their Applications, Dover, New York, 1972.

[12] P.-L. Lions, M. Musiela, Correlations and bounds for stochastic volatility models, Ann. Inst. H. Poincaré Anal. Non Linéaire 24 (2007), 1-16.

[13] H. Matsumoto, M. Yor, Exponential functionals of Brownian motion, I. Probability laws at fixed time, Probab. Surv. 2 (2005), 312-347.

[14] R. Rebonato, Volatility and Correlation. The Perfect Hedger and the Fox, second ed., Wiley, Chichester, 2004.

[15] D. Revuz, M. Yor, Continous Martingales and Brownian Motion, third ed. Grundlehren Math. Wiss. 293, Springer, Berlin, 2005.

[16] C. Sin, Complications with stochastic volatility models, Adv. in Appl. Probab. 30 (1998), 256-268. 
